\title{
Adsorção em coluna de leito fixo aplicada para a pré- concentração de cádmio em amostras de água
}

Fixed bed column adsorption applied to the preconcentration of cadmium in water samples

\author{
H. A. Silva Neto ${ }^{1 *}$; H. L. Garcia ${ }^{1}$; R. G. O. Araujo²; C. A. B. Garcia ${ }^{3}$ \\ ${ }^{1}$ Departamento de Engenharia Química/Laboratório de Operações Unitárias, Universidade Federal de Sergipe, \\ 49.100-000, São Cristóvão-Sergipe, Brasil \\ ${ }^{2}$ Instituto de Química/Laboratório de Estudos em Química Analítica Ambiental, Universidade Federal de Bahia, \\ 40.170-115, Salvador-Bahia, Brasil \\ ${ }^{3}$ Departamento de Química/Laboratório de Química Analítica Ambiental, Universidade Federal de Sergipe, \\ 49.100-000, São Cristóvão-Sergipe, Brasil
}

*humberto.qi@hotmail.com.br

(Recebido em 25 de julho de 2017; aceito em 24 de maio de 2018)

\begin{abstract}
Neste trabalho foi aplicado um processo de adsorção para pré-concentração de cádmio complexado pelo composto orgânico 1-(2-piridilazo)-2-naftol (PAN) em pH 11-12, sendo o adsorvente carvão ativado empregado como meio sólido empacotado em coluna. Foi realizada a caraterização do material adsorvente carvão, que apresentou ponto de carga zero na faixa de $\mathrm{pH} 8$ e 10. Foi possível avaliar também a presença de grupos funcionais como hidroxilas, carbonila, anéis aromáticos e álcoois. O modelo da isoterma de Freundlich foi ajustado aos dados experimentais de equilíbrio por estimação não linear utilizando um programa computacional. A adsorção do complexo $\mathrm{Cd}(\mathrm{PAN})_{2}$ em coluna de carvão ativado com alimentação de $2 \mathrm{mg} \mathrm{L}^{-1}$ de $\mathrm{Cd}^{2+}$ apresentou como parâmetros da curva de ruptura o tempo útil e total de 6,20 e 25 minutos, respectivamente. A capacidade de adsorção de $\mathrm{Cd}^{2+} \mathrm{em}$ carvão foi $0,6250 \mathrm{mg} \mathrm{g}^{-1}$, e altura útil da coluna foi de $1,24 \mathrm{~cm}$. Foi otimizado um método para pré-concentração de $\mathrm{Cd}^{2+}$ empregando a espectrometria de absorção atômica com chama (FAAS). O fator de enriquecimento foi de 32 vezes. $\mathrm{O}$ método de pré-concentração foi aplicado para a determinação de $\mathrm{Cd}^{2+} \mathrm{em}$ amostras de resíduo laboratorial e de água torneira. O método de enriquecimento apresentou-se viável para o enriquecimento das amostras, com um o tempo de preparação da amostra inferior a 15 minutos.

Palavras-chave: Adsorção, Pré-concentração, Cádmio.
\end{abstract}

In this work an adsorption process was applied to the preconcentration of cadmium complexed by organic compound 1-(2-pyridylazo)-2-naphthol (PAN) in pH 11-12, the adsorbent activated carbon, being used as solid medium packed in column. Characterization of the charcoal adsorbent material was carried out, with a zero load point in the range of $\mathrm{pH} 8$ and 10. It was also possible to evaluate the presence of functional groups such as hydroxyl, carbonyl, aromatic rings and alcohols. The Freundlich isotherm model was fitted to the equilibrium experimental data by nonlinear estimation using computational software. The adsorption of the $\mathrm{Cd}(\mathrm{PAN})_{2}$ complex in activated carbon column with $2 \mathrm{mg} \mathrm{L}^{-1}$ of $\mathrm{Cd}^{2+}$ presented as parameters of the breakdown curve the useful and total time of 6.20 and 25 minutes, respectively. The adsorption capacity of $\mathrm{Cd}^{2+}$ in coal was $0.6250 \mathrm{mg} \mathrm{g}^{-1}$, and useful column height was $1.24 \mathrm{~cm}$. A method for preconcentration of $\mathrm{Cd}^{2+}$ was optimized using flame atomic absorption spectrometry (FAAS). The enrichment factor was 32 times. The preconcentration method was applied for the determination of $\mathrm{Cd}^{2+}$ in samples of laboratory residue and tap water. The enrichment method was feasible for the enrichment of the samples, with a sample preparation time of less than 15 minutes.

Keywords: Adsorption, preconcentration, cadmium.

\section{INTRODUÇÃO}

A preocupação mundial com a preservação ambiental vem aumentando nas últimas décadas, essa maior atenção é fundamental, pois existe um crescimento da população e do consumo de bens industrializados. É necessário o surgimento e fortalecimento das políticas de preservação ambiental, a fim de estabelecer um equilíbrio, como também trabalhar uma educação ambiental.

A fonte da poluição ambiental está centralizada nas atividades antropogênicas, ação humana, que são desenvolvidas no cotidiano das cidades, como a emissão de poluentes provenientes das indústrias, da agricultura, e do tráfego de automotores, entretanto existe uma contribuição da 
poluição originada em processos que contemplam a ação da natureza, como o intemperismo, a erosão de depósitos naturais, a erupção vulcânica e emissão de gases [1,2].

Dentre os diferentes tipos de poluentes, os metais tóxicos apresentam grande ameaça, na maioria dos estudos esses metais são encontrados em baixos níveis de concentrações, porém o seu efeito acumulativo e/ou a baixa taxa de eliminação pelo corpo humano agravam as consequências [3].

Os estudos para avaliação da contaminação do meio, muita das vezes requer um monitoramento mais efetivo do local, métodos de especiação adequado, pois na maioria dos casos, os níveis de concentração dos contaminantes encontram-se abaixo dos limites de detecção das técnicas analíticas. Sendo necessária, uma amostragem e extração adequada desses contaminantes, uma quantificação por métodos analíticos que apresentam baixo limite de detecção, elevada seletividade e baixo custo [4].

\subsection{Metais traço}

Alguns metais traço são agentes tóxicos, causadores de inúmeros efeitos adversos para a saúde humana. No Japão, em 1960, aconteceu um surto de uma doença originada da contaminação por cádmio, a doença óssea itai-itai [2].

Em 2013, existiu um alerta para a possível contaminação por Cd presente na composição das bijuterias, que são importadas da China para o Brasil. Atualmente, as principais fontes de liberação de $\mathrm{Cd}$ são os efluentes e emissões das siderúrgicas, a corrosão de tubos galvanizados, a fabricação de fertilizantes fosfatados, a reciclagem do $\mathrm{Cd}$ em sucata, a fabricação de pigmentos, a impressão na indústria fotográfica, o uso do lodo de esgoto na agricultura, há também a contribuição por ação natural, como o intemperismo e a erosão de depósitos naturais de cádmio $[1,2,3]$.

O corpo humano não utiliza o $\mathrm{Cd}$ em nenhuma das suas necessidades fisiológicas, ou seja, este metal é altamente tóxico para a saúde do homem, mesmo em níveis baixos de concentrações, geralmente a contaminação ocorre por via oral e seus efeitos tóxicos são muito agressivos. Essas complicações são mais acentuadas para os rins, ocasionando o comprometimento de outros órgãos, a desmineralização e danos aos ossos [1,2].

\subsection{Legislações Ambientais}

Para manter e alcançar uma segurança ambiental frente aos contaminantes ambientais, os países e, até mesmo, os blocos econômicos como o mercado comum do sul (Mercosul) estabelecem diretrizes, tratados, protocolos e normas com o objetivo de atingir uma unificação quanto aos limites máximos dos contaminantes. A legislação brasileira com o uso da Portaria $\mathrm{n}^{\circ}$ 2914, de dezembro de 2011, estabelece o valor máximo permitido de Cd em água potável $(0,005$ $\mathrm{mg} \mathrm{L}^{-1}$ ), esse mesmo limite também é estabelecido pela agência de proteção ambiental dos Estados Unidos [5,6,7]. Quanto às águas de descarte, a legislação brasileira com o uso da Resolução - RDC n 430, de 13 de maio de 2011, estabelece o valor máximo permitido de cádmio de $0,2 \mathrm{mg} \mathrm{L}^{-1}$, regulamentado pelo Conselho Nacional de Meio Ambiente [8]. O Cd faz parte da lista de substâncias tóxicas, elaborada pela agência de substâncias tóxicas e registro de doenças $[9,10]$.

\subsection{Pré-concentração}

As técnicas de pré-concentração têm como finalidade o enriquecimento do analito nas amostras, a adsorção em carvão ativo apresenta-se como uma alternativa possível para aplicação [11]. A complexação dos íons de metais é uma etapa fundamental para o enriquecimento do analito nas amostras, pois os íons serão isolados de maneira mais fácil e eficiente [2,12].

Diferentes agentes ligantes são utilizados, o complexante 1-(2-piridilazo)-2-naftol, (PAN), proporciona a complexação de alguns íons metálicos, entres eles o $\mathrm{Cu}^{2+}, \mathrm{Ni}^{2+}, \mathrm{Zn}^{2+}, \mathrm{Co}^{2+}, \mathrm{Mn}^{2+} \mathrm{e}$ os íons de metais tóxicos, como o $\mathrm{Cd}^{2+}$ e o $\mathrm{Hg}^{2+}$. O PAN reage de maneira muito sensível e 
seletiva, como consequência dessa reação há a formação de quelatos insolúveis no meio aquoso [13].

\subsection{Processo de Adsorção}

A adsorção é um processo físico-químico de interação superficial entre o meio adsorvente e o adsorvato, no qual uma mistura multicomponente de fluido (gás ou líquido), adsorvato, é atraída para a superfície de um adsorvente sólido, formando ligações através de interações físicas ou químicas, sendo que as moléculas adsorvidas se encontram retidas na superfície do adsorvente [14].

O carvão ativado é muito utilizado como adsorvente e apresenta notável destaque para essa aplicação, uma vez que, suas características como porosidade, área superficial, grupos funcionais, estabilidade térmica, baixa reatividade com ácidos e bases, possibilita uma maior capacidade de adsorção e versatilidade na remoção de poluentes orgânicos e inorgânicos, estejam eles dissolvidos em meio aquoso ou gasoso [14].

\subsection{Equilíbrio de Adsorção}

Nos estudos do processo de adsorção é essencial estabelecer o modelo de isoterma de adsorção mais adequado. A curva de uma isoterma de adsorção descreve o fenômeno que regula a mobilidade de uma substância em relação ao meio poroso. A condição de equilíbrio do processo é alcançada, quando as concentrações de adsorvato na superfície do adsorvente e na solução atinge o equilíbrio dinâmico. A Equação 1 representa a quantidade de adsorvato no adsorvente em equilíbrio [14].

$$
\begin{aligned}
& Q_{E q} \\
& =\frac{V\left(\mathrm{C}_{0}-\mathrm{C}_{E q}\right)}{m_{a d s}}
\end{aligned}
$$

em que: $\mathrm{Q}_{\mathrm{Eq}}$ é a quantidade adsorvida por unidade de massa do adsorvente em equilíbrio (mg g$\left.{ }^{1}\right), \mathrm{C}_{0}$ é a concentração inicial da solução de adsorvato $\left(\mathrm{mg} \mathrm{L}^{-1}\right), \mathrm{C}_{\mathrm{Eq}}$ é a concentração de adsorvato na solução no equilíbrio $\left(\mathrm{mg} \mathrm{L}^{-1}\right), \mathrm{V}$ é o volume da solução $(\mathrm{L})$ e $\mathrm{m}$ é a massa seca de adsorvente (g).

\subsection{Isoterma de Langmuir}

O modelo da isoterma de Langmuir tem sua origem no estudo de adsorção gás e sólido. Esta isoterma é um modelo empírico, em que assume os seguintes pressupostos: a adsorção ocorre em monocamadas, somente acontece a adsorção em um número fixo de locais definidos, e esses locais são idênticos e equivalentes, nos quais não há interação lateral nem estérica entre as moléculas adsorvidas, as quais estão situadas próxima ou ao lado das outras. Este modelo caracteriza um processo de adsorção homogêneo, pois cada molécula possui entalpia e energia constante, ou seja, não existe diferença de afinidade entre os sítios ativos e o adsorvato. A Equação 2 representa o modelo de Langmuir [14].

$$
Q_{E q}=\frac{Q_{M a x} \cdot b \cdot C_{E q}}{1+b \cdot C_{E q}}
$$

em que, QMax é a concentração máxima do adsorvato adsorvido no material sólido ( $\left.\mathrm{mg} \mathrm{g}^{-1}\right)$, e b é a constante de equilíbrio de adsorção de Langmuir $\left(\mathrm{L}_{\mathrm{mg}} \mathrm{mg}^{-1}\right)$, os significados físicos para as constantes desse modelo são: b representa a razão entre a taxa de adsorção e a taxa de dessorção, ou seja, elevados valores de b é um indicativo de forte afinidade do adsorvato pelos sítios ativos do adsorvente, QMax representa a quantidade de sítios ativos disponíveis no material adsorvente. 


\subsection{Isoterma de Freundlich}

A isoterma de Freundlich é uma relação, que descreve o processo de adsorção não ideal e reversível, onde não há a restrição de formação da monocamada. O modelo possibilita os estudos de adsorção em camadas múltiplas, nas quais existe uma distribuição não uniforme do calor de adsorção e da afinidade sobre a superfície heterogênea.

O modelo de Freundlich é muito aplicado em sistemas heterogêneos com destaque para adsorção de compostos orgânicos ou espécies de alta afinidade com o carvão ativado. A Equação 3 representa o modelo de Freundlich [14].

$$
Q_{E q}=k_{f} \cdot C_{E q}^{n}
$$

em que, $K_{f}$ e $n$ são as constantes empíricas relacionadas ao sistema adsorvente e soluto do modelo de Freundlich. Os significados físicos para as constantes desse modelo são: $n$ representa a intensidade do processo de adsorção ou heterogeneidade da superfície. Considerando $n<1$ para uma quimiossorção, e valores de $n>1$ uma fisissorção, o significado físico de $K_{f}$ é uma medida aproximada da capacidade de adsorção do adsorvente, em que, quanto maior o valor de $K_{f}$ maior a sua capacidade de adsorção, entretanto o modelo de Freundlich é limitado para baixas concentrações [14].

\subsection{Adsorção em Leito Fixo}

A adsorção de poluentes tóxicos em colunas de leito fixo, depende das características físicas e químicas do material adsorvente e, também, das características do processo com aplicação na pré-concentração e determinação de poluentes ambientais [11,15]

Para a utilização de uma coluna é necessário que a mesma apresente um bom desempenho, ou resultados satisfatórios. O seu desempenho é avaliado comumente com os estudos de curva de ruptura ou curva de breakthrough, em que é possível predizer o ponto de saturação da coluna de adsorção mediante as condições de alimentação. A curva de ruptura é uma relação entre a razão da concentração de saída $\left(C_{\mathrm{s}}\right)$ pela concentração da alimentação $\left(\mathrm{C}_{\mathrm{e}}\right)$, e o tempo, como é mostrado na Figura $1[16]$.

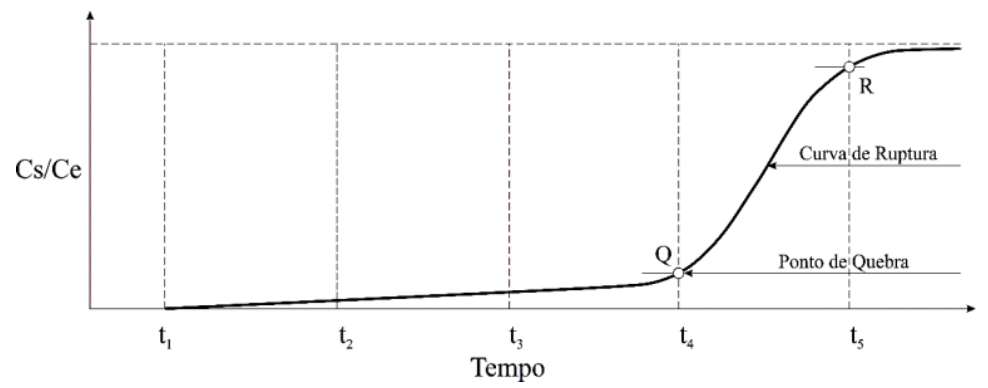

Figura 1: Curva de Ruptura em coluna de Leito Fixo Fonte: Adaptada de Geankoplis ( 2003)

Experimentalmente é fácil a obtenção da curva de ruptura. A partir da passagem da solução de adsorvato pela coluna empacotada com o material adsorvente, e o monitoramento da concentração na saída da coluna. A razão $C_{s} / C_{e}$ em função do tempo representa uma curva típica de ruptura da coluna de leito fixo [16].

Com a obtenção da curva de ruptura no ponto de equilíbrio é possível determinar os seguintes parâmetros encontrados na Tabela $1[17,18,19]$.

Na Tabela 1 encontram-se os parâmetros da coluna de adsorção, que são representados pelas Equações (4), (5), (6) e (7). O tempo total e o útil do processo de adsorção em coluna de leito fixo é dado em segundos (s), o tempo total é definido como proporcional a capacidade de máxima de adsorção da coluna, entretanto o tempo útil é definido como o instante percorrido até o ponto de ruptura $\left(t_{b}, C_{b}\right)$, em que $t_{b}$ e $C_{b}$ representam o tempo e a concentração em mg. $\mathrm{L}^{-1}$ no ponto de quebra, respectivamente. $C_{e}$ é a concentração de adsorvente na alimentação [19]. 
Tabela 1: Determinação dos parâmetros da coluna de leito fixo

$\begin{array}{ll}\text { Parâmetros da coluna de adsorção } & \text { Equação } \\ \text { Tempo total } & t_{t}=\int_{0}^{t_{\infty}}\left(1-\frac{C_{s}}{C_{e}}\right) d t\end{array}$

Tempo útil

$$
t_{u}=\int_{0}^{t b}\left(1-\frac{C_{b}}{C_{e}}\right) d t
$$

$$
\begin{array}{ll}
\text { Altura útil } & H_{u}=\frac{t_{u}}{t_{t}} H_{t} \\
\hline \text { Capacidade de Saturação } & Q_{E q}=\frac{C_{\mathrm{e}} \cdot Q}{m_{\text {ads }}} t_{t}
\end{array}
$$

A altura total e a altura útil representam a altura total de empacotamento da coluna com o material adsorvente em $\mathrm{cm}$, e a altura utilizada até o ponto de ruptura do leito $\mathrm{em} \mathrm{cm}$, respectivamente. A capacidade de adsorção por massa de material adsorvente em $\mathrm{mg} \mathrm{g}^{-1}$ é representada pela Equação $7, Q$ é a vazão volumétrica da alimentação em $\mathrm{mL} \cdot \mathrm{min}^{-1}$ e $\mathrm{m}_{\mathrm{ads}}$ é a massa de adsorvente em g [19].

$\mathrm{Na}$ adsorção existe a transferência de massa da fase fluida para a fase sólida, o inverso desse processo de transferência de massa é a dessorção, pois ela tem como finalidade a regeneração do material adsorvente, mas também a recuperação do adsorvato, além disso o adsorvato pode ser uma substância tóxica, e necessitar de um encaminhamento e condicionamento mais adequado ocupando um menor volume. O adsorvato também pode ser um analito, ou um produto e será necessário dar continuidade em outros procedimentos como na determinação analítica, e purificação, uma vez que uma etapa de separação já foi realizada [16].

Neste contexto, o presente trabalho tem como objetivo a aplicação de um método analítico para a determinação do metal pesado cádmio, utilizando uma coluna de carvão ativo para a préconcentração de cádmio, e aplicar este método em matriz de contaminação, a fim de avaliar a disponibilidade do metal.

\section{MATERIAL E MÉTODOS}

\subsection{Reagentes e Amostras}

Os reagentes químicos utilizados apresentam grau analítico. Como material adsorvente foi utilizado carvão vegetal ativado, com faixa granulométrica de 20/40 mesh. As amostras de resíduo líquido e de água foram coletadas no Laboratório de Química Analítica Ambiental, do Departamento de Química, na Universidade Federal de Sergipe.

\subsection{Instrumentação}

Foram utilizados agitador magnético (Solab), incubadora de bancada (Cientec), balança analítica (Shimadzu AY 220), medidor de pH digital (Az), paquímetro (Messen), bomba peristáltica (Gilson minipuls 3), espectrômetro de infravermelho (Shimadzu IR Restrige 21), espectrômetro de absorção atômica com chama (Shimadzu AAS-7000) no comprimento de 228,2 $\mathrm{nm}$ e fenda de $0,8 \mathrm{~nm}$.

\subsection{Caracterização do Material Adsorvente}

A determinação do ponto de carga zero PCZ do adsorvente foi seguido o método de Boehm. A determinação dos grupos funcionais foi feita por espectrometria de infravermelho com 
transformada de Fourier (FTIR, Shimadzu IR Restrige 21). O carvão ativado caracterizado e utilizado como adsorvente é o mesmo material, que é aplicado como adsorvente na bancada de adsorção para sistema sólido-líquido, no Laboratório de Operações Unitárias do Departamento de Engenharia Química, na Universidade Federal de Sergipe.

\subsection{Montagem da Coluna de Leito Fixo}

A minicoluna de leito fixo foi montada a partir de um tubo de polietileno, com o comprimento de $9 \mathrm{~cm}$, e diâmetro interno de $4 \mathrm{~mm}$. Foi feito um empacotamento com 399,4 $\mathrm{mg}$ de material adsorvente, como mostra a Figura 2.

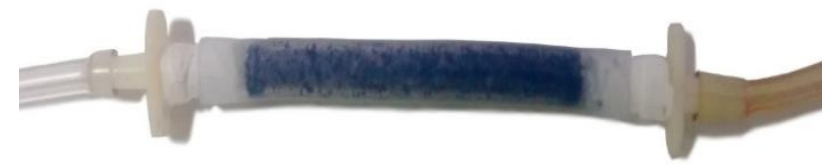

Figura 2: Coluna de adsorção com carvão ativado

\subsection{Isoterma de Adsorção}

O procedimento para a determinação da isoterma do processo de adsorção foi realizado em triplicata. A adsorção, em batelada, de $100 \mathrm{~mL}$ de solução de Cd (II), na faixa de concentração de $0,5 \mathrm{mgL}^{-1}$ a $5 \mathrm{mgL}^{-1}$, complexada por PAN, e usando $1,0 \mathrm{~g}$ de carvão vegetal ativado. Em $\mathrm{pH}$ inicial do meio igual a 12 , corrigido com $\mathrm{NaOH}\left(1 \mathrm{~mol} \mathrm{~L}^{-1}\right)$, em temperatura de $25^{\circ} \mathrm{C}$, e agitação em $150 \mathrm{rpm}$. Houve a coleta de $6,0 \mathrm{~mL}$ do sobrenadante após 22 horas de experimentos, o analito foi determinado por espectrometria de absorção atômica com chama.

\subsection{Curva de Ruptura da Coluna de Leito Fixo}

A curva de ruptura foi realizada a partir do procedimento experimental de adsorção na coluna de leito até atingir a sua saturação. A saturação foi atingida mediante a passagem da solução de $\mathrm{Cd}^{2+}\left(2 \mathrm{mg} \mathrm{L}^{-1}\right)$ complexada por $1,76 \mathrm{~mL}$ de PAN (500 $\left.\mathrm{mg} \mathrm{L}^{-1}\right)$, em coluna de carvão, com pH igual a 12 corrigido com $\mathrm{NaOH}\left(5 \mathrm{~mol} \mathrm{~L}^{-1}\right)$. A vazão volumétrica da alimentação foi de $5 \mathrm{~mL}$ $\min ^{-1}$. Houve a coleta do efluente a cada 5 minutos, e o analito presente no efluente foi determinado por FAAS.

\subsection{Curvas de calibração e Amostras}

Houve a preparação de curvas de calibração, uma curva baseada no experimento de préconcentração com a coluna de adsorção seguindo metodologia semelhante da saturação da coluna, porém foram eluídas em fluxo oposto com $3,0 \mathrm{~mL}$ de $\mathrm{HCl} \mathrm{1,0} \mathrm{mol} \mathrm{L}^{-1}$, e a outra curva sem o procedimento de pré-concentração, a faixa crescente de concentração na curva foi de 10 a $200 \mu \mathrm{g}$ $\mathrm{L}^{-1}$ de $\mathrm{Cd}^{2+}$.

A determinação de $\mathrm{Cd}^{2+}$ nas amostras foram em triplicatas, em que de $50 \mathrm{~mL}$ da amostra seguindo com o ajuste do $\mathrm{pH}$ do meio a cerca de 11-12 com NaOH 1,0 mol L-1, complexada com o agente PAN, após complexação a amostra foi adsorvida em coluna de carvão ativado, seguida de eluição em fluxo oposto com $3,0 \mathrm{~mL}$ de $\mathrm{HCl} \mathrm{1,0} \mathrm{mol} \mathrm{L}^{-1}$.

\section{RESULTADOS E DISCUSSÃO}

A caracterização do ponto de carga zero (PCZ) e dos grupos funcionais presentes na superfície do material adsorvente está representada nas Figuras 3 e 4, respectivamente. 


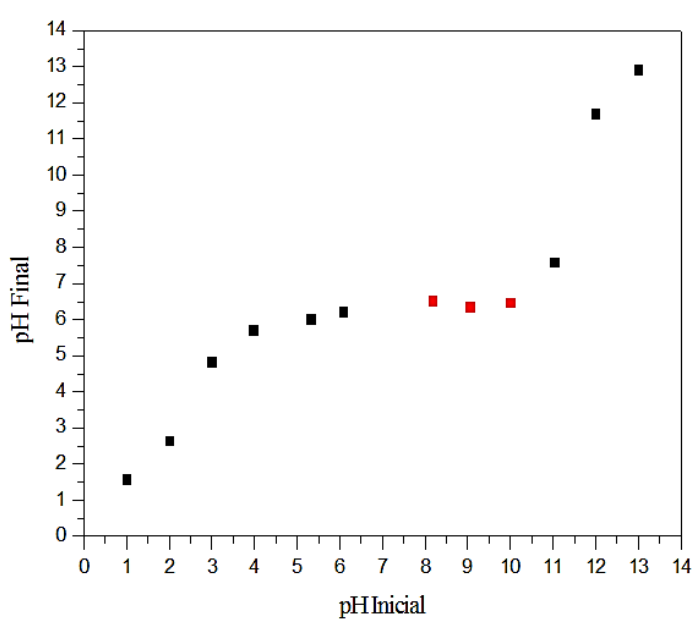

Figura 3: Ponto de carga Zero (PCZ)

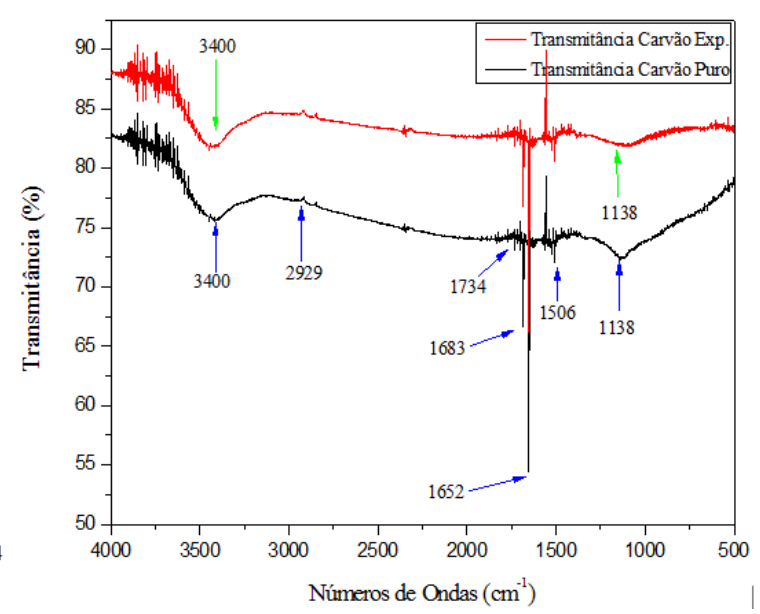

Figura 4: Determinação dos grupos funcionais

O PCZ é uma avaliação oriunda do pH do meio e a influência dos grupos funcionais presentes na superfície do material. Este estudo tem a finalidade de definir em que condição de $\mathrm{pH}$ a carga total superficial é nula. Na Figura 3 é possível observar que na faixa de $\mathrm{pH}$ inicial da solução $(8<$ pH pcz <10) é encontrada a região de PCZ, funcionado como região de tampão, entretanto para valores de $\mathrm{pH}$ acima de 10 e abaixo de 6 são mais representativos para uma região de basicidade e acidez, respectivamente.

No espectro de infravermelho, representado pela Figura 4, encontra-se as transmitâncias do carvão experimental (Carvão Exp.), sendo este caraterizado após o processo de adsorção, e o carvão puro (Carvão Puro), que foi caracterizado entes do processo de adsorção. É possível observar a banda de $3400 \mathrm{~cm}^{-1}$, característica do estiramento vibracional de grupo $-\mathrm{OH}$, as vibrações em torno de $2929 \mathrm{~cm}^{-1}$ representam resíduos do desaparecimento de ligações C-H. Os picos por volta de $1734 \mathrm{~cm}^{-1}$ e $1652 \mathrm{~cm}^{-1}$ representam a presença de grupos carbonila $\mathrm{C}=\mathrm{O}$. A presença de picos em aproximadamente $1600 \mathrm{~cm}^{-1}$ até $1400 \mathrm{~cm}^{-1}$, são característicos do estiramento de ligações $\mathrm{C}-\mathrm{C}$ e $\mathrm{C}=\mathrm{C}$ dos anéis aromáticos ou anéis em lignina [20,21,22]. A banda em torno de $1038 \mathrm{~cm}^{-1}$ representa ligações $\mathrm{C}-\mathrm{O}$ de álcoois. Prosseguindo com a análise foi possível também observar modificação nos picos, e o desaparecimento da banda por volta de 1138 $\mathrm{cm}^{-1}$, sendo esta banda característica das ligações C-O dos álcoois, evidenciando uma maior interação com o complexo.

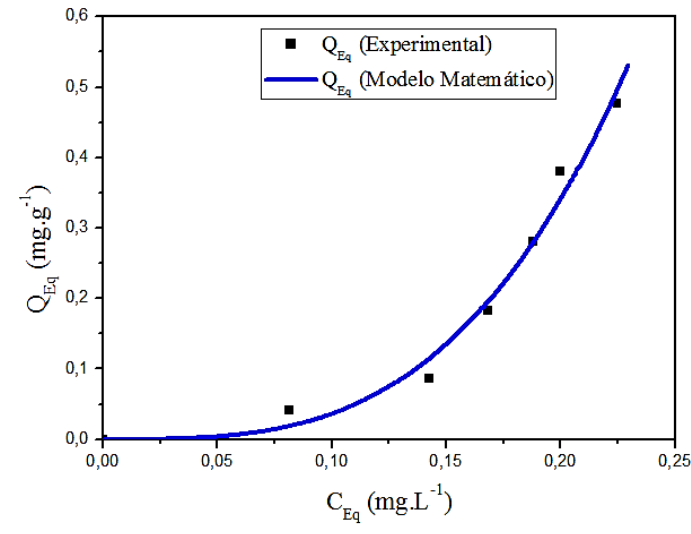

Figura 5: Isoterma de Equilíbrio

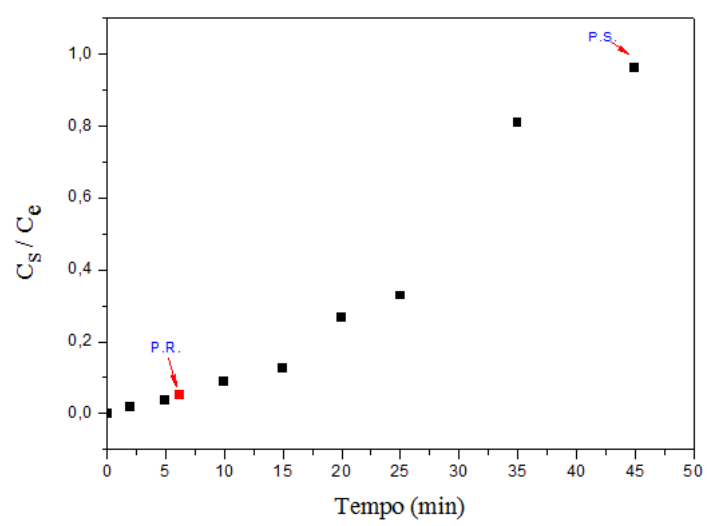

Figura 6: Curva de ruptura da Coluna

A isoterma de equilíbrio foi avaliada a partir da estimação dos parâmetros de forma não linear, a fim de obter uma melhor definição para a isoterma do processo, e um modelo matemático que se adeque aos dados experimentais. Dentre o modelo Langmuir e Freundlich, o modelo que melhor representou os dados experimentais foi o modelo de Freundlich, ficando evidente quando comparado o perfil da curva experimental com os dados do modelo matemático. Para a estimação dos parâmetros de Freundlich $\mathrm{K}_{f}$ e $n$, foram utilizados algoritmos de estimação. Como resultado da estimação não linear foi possível definir os parâmetros empíricos, $\mathrm{K}_{f}$ igual a $60,80 \mathrm{mg} \cdot \mathrm{g}^{-1} \mathrm{e} n$ 
igual a 3,22. Com isso o modelo de Freundlich ficou definido como $\mathrm{Q}_{\mathrm{Eq}}=60,80 . \mathrm{C}_{\mathrm{Eq}}^{3,22}$, sendo a função objetivo erro quadrado $0,003402 \mathrm{mg}^{2} \mathrm{~g}^{-2}$, a Figura 5 representa o modelo de Freundlich.

A coluna de adsorção apresentou o comportamento de ruptura mostrado na Figura 6, em que os pontos (PR) e (PS) representam o ponto de ruptura e o ponto de saturação da coluna, respectivamente. $O$ ponto de ruptura foi calculado considerando a concentração de saída da coluna sendo igual a $5 \%$ da concentração de alimentação da coluna, com isso o tempo de ruptura foi de 6,20 minutos, e 25 minutos o tempo de saturação da coluna de carvão ativado como mostra a Tabela 2 [19].

Tabela 2: Parâmetros da coluna de pré-concentração de cádmio

\begin{tabular}{lccccc}
$\mathbf{C}_{\mathbf{0}}\left(\mathbf{m g ~ L}^{-\mathbf{1}}\right)$ & $\mathbf{Q}\left(\mathbf{m L} \mathbf{m i n}^{-\mathbf{1}}\right)$ & $\mathbf{t}_{\mathbf{u}}(\mathbf{m i n})$ & $\mathbf{t}_{\mathbf{t}}(\mathbf{m i n})$ & $\mathbf{H}_{\mathbf{u}}(\mathbf{c m})$ & $\mathbf{Q E q}\left(\mathbf{m g ~ g}^{-\mathbf{1}}\right)$ \\
\hline $2,0 \mathrm{mg} \mathrm{L}^{-1}{\mathrm{de} \mathrm{Cd}^{2+}}^{5}$ & 6,20 & 25 & 1,24 & 0,625
\end{tabular}

O método de enriquecimento do analito é mais instável e menos ajustável pela curva linear, pois a montagem da curva pré-concentrada dependerá da complexação dos íons de $\mathrm{Cd}$, da adsorção e dessorção, e também da estabilidade da coluna. $\mathrm{O}$ valor do fator de pré-concentração foi calculado pela razão entre coeficiente angular da curva calibração sem pré-concentração (Abs

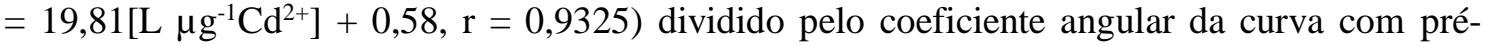
concentração (Abs $\left.=0,62\left[\mathrm{~L} \mathrm{\mu} \mathrm{g}^{-1} \mathrm{Cd}^{2+}\right], \mathrm{r}=0,9991\right)$, sendo igual a 32 vezes.

Na Tabela 3 encontra a aplicação do método de pré-concentração do analito, através da análise de três diferentes amostras e em triplicatas. Na amostra de água da torneira não foi possível determinar a presença de íons de cádmio pela técnica aplicada, entretanto, foi possível determinar a presença de cádmio nas amostras de resíduo do laboratório e de água da torneira.

Tabela 3: Aplicação do método de pré-concentração de cádmio em amostras reais

\begin{tabular}{lc}
\hline Amostra & Concentração de $\mathbf{C d}^{2+}\left(\mathbf{m g ~ L}^{-\mathbf{1}}\right)$ \\
\hline Água da torneira & N.D. \\
\hline Resíduo do laboratório & $0,161 \pm 0,002$ \\
\hline * Não Determinado &
\end{tabular}

$\mathrm{Na}$ amostra de resíduo do laboratório a concentração determinada foi de $0,161 \pm 0,002 \mathrm{mg} \mathrm{L}$ ${ }^{1}$ estando dentro do limite máximo permitido para o descarte direto em corpo receptor definido pela Resolução $n^{\circ} 430$ do COMANA.

\section{CONCLUSÃO}

Neste trabalho foi realizado a aplicação de um método de pré-concentração, para a determinação de $\mathrm{Cd}^{2+}$ em amostras de água. Para quantificar e qualificar, caracterizar, o método de pré-concentração, que utiliza o processo de adsorção em coluna de leito fixo, foi realizada a caraterização do material adsorvente, com a determinação dos grupos funcionais na superfície do carvão por FTIR e a determinação do ponto de carga zero, que está compreendido dentro da faixa de $\mathrm{pH}$ de 8 até 10.

Os estudos do equilibro de adsorção foram realizados em batelada, em que dados de equilíbrio foram melhores representados pelo modelo de isoterma de Freundlich não linearizada. A isoterma ficou representada por $\mathrm{Q}_{\mathrm{Eq}}=60,80 . \mathrm{C}_{\mathrm{Eq}}^{3,22}$, sendo a função objetivo erro quadrado igual a $0,003402 \mathrm{mg}^{2} \mathrm{~g}^{-2}$. Entretanto, o estudo do processo de adsorção em coluna de leito fixo foi baseado na determinação da curva de ruptura, curva de breakthrough, e foram definidos os seguintes parâmetros para esse estudo, $\mathrm{C}_{0}=2 \mathrm{mg} \cdot \mathrm{L}^{-1} \mathrm{de} \mathrm{Cd}^{2+}, \mathrm{Q}=5 \mathrm{~mL} \mathrm{~min}{ }^{-1}, \mathrm{t}_{\mathrm{u}}=6,20 \mathrm{~min}, \mathrm{t}_{\mathrm{t}}=$ $25 \mathrm{~min}, \mathrm{H}_{\mathrm{u}}=1,24 \mathrm{~cm}$ e $\mathrm{Q}_{\mathrm{Eq}}=0,625 \mathrm{mg} \mathrm{g}^{-1}$, em que a massa de $0,400 \mathrm{~g}$ do adsorvente apresentou um bom tempo de saturação. A avaliação do método de pré-concentração de $\mathrm{Cd}$ determinou um fator de enriquecimento de 32 vezes, quando comparado com uma determinação sem a préconcentração da amostra. 
Em fim, o método de pré-concentração que utilizou uma coluna de adsorção empacotada com carvão ativado apresentou-se como uma metodologia viável para o enriquecimento do metal pesado $\mathrm{Cd}^{2+}$, em amostras de água, e com possível aplicação em uma rotina laboratorial de quantificação, pois a coluna utiliza uma baixa quantidade de material adsorvente, $0,400 \mathrm{~g}$, sendo possível a regeneração do adsorvente da coluna por eluição do adsorvato, entretanto alguns ajustes para evitar a formação de bolhas de ar no interior da coluna e um melhor controle da temperatura do processo, reduzirá os erros no método de pré-concentração.

\section{AGRADECIMENTOS}

Este trabalho foi apoiado pelo Conselho Nacional de Desenvolvimento Científico e Tecnológico (CNPq) e Fundação de Amparo à Pesquisa do Estado da Bahia (FAPESB), sob a forma de bolsas de pesquisa e auxílio financeiro.

\section{REFERÊNCIAS BIBLIOGRÁFICAS}

1. Vignola F, Borges D, Curtius A, Welz B, Becker-Ross H. Simultaneous determination of Cd and Fe in sewage sludge by high-resolution continuum source electrothermal atomic absorption spectrometry with slurry sampling. Microchemical Journal. 2010;95:333-336, doi:10.1016/j.microc.2010.01.014

2. Purkayastha D, Mishra U, Biswas S. A comprehensive reviw on Cd(II) removal from aqueous solution. Journal of Water Process Engineering. 2014;2:105-128, doi:10.1016/j.jwpe.2014.05.009

3. Baird C, Cann MC. Química ambiental. 4th ed. Porto Alegre, RS: Bookman; 2011.

4. Bisinoti M, Jardim WdF. O emprego de técnicas analíticas na especiação de metais pesados e a sua importância para o estudo do ambiente. São Paulo: UNICAMP, Departamento de Química Analítica; 2004.

5. Bureau of Indian Standards (BIS). Site Bureau of Indian Standards. [Online].; 2009 [cited 2015 Julho 24. Available from: http://bis.org.in/sf/fad/FAD25(2047)C.pdf.

6. Ministério da Saúde. Site do Ministério da saúde. [Online].; 2011 [cited 2015 Julho 23. Available from: http://bvsms.saude.gov.br/bvs/saudelegis/gm/2011/prt2914_12_12_2011.html.

7. US EPA. Site da United States Evironmental Protection Agency. [Online].; 2009 [cited 2015 Julho 23. Available from: http://water.epa.gov/drink/contaminants/basicinformation/cadmium.cfm.

8. Ministério do meio ambiente. Site do ministério do meio ambiente. [Online].; 2011 [cited 2015 Julho 28. Available from: http://www.mma.gov.br/port/conama/legiabre.cfm?codlegi=646.

9. ATSDR. Site da Agency for Toxic Substances and Disease Registry. [Online].; 2008 [cited 2015 Julho 23. Available from: http://www.atsdr.cdc.gov/toxfaqs/tf.asp?id=47\&tid=15.

10. US EPA. Site da United States Evironmental Protection Agency: Integrated Risk Information System (IRIS). [Online].; 1991 [cited 2015 Julho 29. Available from: http://www.epa.gov/iris/subst/0141.htm\#oralrfd.

11 Almeida Neto AFdA, Vieira MGA, Silva MGCd. Adsorption and desorption processes for copper removal from water using different eluents and calcined clay as adsorbent. Journal of Water Process Engineering. 2014;3:90-97, doi:10.1016/j.jwpe.2014.05.014

12. Harris DC. Titulações com EDTA. In HARRIS DC. Química Analítica Quantitativa. 7th ed. Rio de Janeiro: LTC; 2008. p. 253-255.

13. Srijaranai S, Autsawaputtanakul W, Santaladchaiyakit Y, Khameng T, Siriraks A, Deming R. Use of 1(2-pyridylazo)-2-naphthol as the post column reagent for ion exchange chromatography of heavy metals in environmental samples. Microchemical Journal. 2011;99:152-158, doi:10.1016/j.microc.2011.04.015

14. Foo K, Hameed B. Review: Insights into the modeling of adsorption isotherm systems. Chemical Engineering Journal. 2010;156:2-10, doi:10.1016/j.cej.2009.09.013

15. Zhou Q, Xing A, Zhao K. Simultaneous determination of nickel, cobalt and mercury ions in water samples by solid phase extraction using multiwalled carbon nanotubes as adsorbent after chelating with sodium diethyldithiocarbamate prior to high performance liquid chromatography. Journal of Chromatography A. 2014;1360:76-81, doi:10.1016/j.chroma.2014.07.084

16. Luz ADd. Aplicação de coluna de adsorção em leito fixo para a remoção de compostos BTX multicomponentes presentes em efluentes petroquímicos. Florianópolis-SC: Tese (doutorado) Universidade Federal de Santa Catarina, Centro Tecnológico. Programa de Pós-Graduação em Engenharia Química; 2012.

17. Barbosa J, Figueredo C, Pereira A, Neto J, Ostroski I. Adsorção do íon cádmio em colunas de leito fixo utilizando o carvão de osso. In X-Encontro brasileiro sobre adsorção; 2014; Guarujá-SP. p. 7. 
18. Bordignon V, Ribeiro C, Borba C, Scheufele F. Obtenção das curvas de ruptura de adsorção do corante reativo Azul 5G utilizando escamas do peixe Oreochromis niloticus. In III Encontro Paranaense de Engenharia e Ciência; 2013; Toledo-PR. p. 7.

19. Geankoplis CJ. Transport processes and separation process principles. 4th ed. New Jersey, Estados Unidos: Prentice Hall; 2003.

20. Brum S, Bianchi M, Silva V, Gonçalves M, Guerreiro M, Oliveira L. Preparação e caracterização de carvão ativado produzido a partir de resíduos do beneficiamento do café. Química Nova. 2008 Jul;31(5):1048-1052, doi:10.1590/S0100-40422008000500019

21. Costa PDd, Furmanski LM, Dominguinic L. Produção, caracterização e aplicação de carvão ativado de casca de nozes para adsorção de azul de metileno. Revista Virtual de Química. 2015 Abr;7(4):12711285, doi:10.5935/1984-6835.20150070

22. Sales P, Bertoli A, Pinto F, Magriotis Z. Produção, caracterização e aplicação do carvão ativado obtido a partir do sabugo de milho: A Busca pelo Reaproveitamento de um Resíduo Agroindustrial. Revista Virtual da Química. 2015 Jan;7(4):1174-1188, doi:10.5935/1984-6835.20150066 